\title{
Molecular to Macroscale Energy Absorption Mechanisms in Biological Body Armour Illuminated by Scanning X-Ray Diffraction with in Situ Compression
}

Yi Zhang, ${ }^{1,2}$ Jan Garrevoet, ${ }^{2}$ Yanhong Wang, ${ }^{3}$ Jan Torben Roeh, ${ }^{2}$ Nicholas J. Terrill, ${ }^{4}$ Gerald Falkenberg, ${ }^{2}$ Yuhui Dong ${ }^{1 *}$ and Himadri S. Gupta ${ }^{3 *}$

1. Institute of High Energy Physics, Chinese Academy of Science, 100049 Beijing, China

2. Deutsches Elektronen-Synchrotron DESY, 22607 Hamburg, Germany

3. Queen Mary University of London, Institute of Bioengineering and School of Engineering and Material Science, E1 4NS London, UK

4. Diamond Light Source, Harwell Science and Innovation Campus, OX11 ODE Harwell, UK

E-mail addresses: zhangyi88@ihep.ac.cn (Yi Zhang), jan.garrevoet@desy.de (Jan Garrevoet), yanhong.wang@qmul.ac.uk (Yanhong Wang), Jan.roeh@desy.de (Jan Torben Roeh), nick.terrill@diamond.ac.uk (Nicholas J. Terrill), gerald.falkenberg@desy.de (Gerald Falkenberg), dongyh@ihep.ac.cn (Yuhui Dong), h.gupta@qmul.ac.uk (Himadri S. Gupta). 


\section{Corresponding authors:}

a): Dr. Himadri S. Gupta

h.gupta@qmul.ac.uk

School of Engineering and Materials Science and Institute of Bioengineering,

Queen Mary University of London, Mile End Road, London E1 4NS, UK

Tel: $+44(0) 2078828867$

Fax: +44(0)207882 3390

b): Prof. Yuhui Dong

Institue of High Energy Physics, Chinese Academy of Sciences

19B Yuquan Road, Shijingshan District, 100049 Beijing, China

dongyh@ihep.ac.cn

Tel: $+86(0) 1088233090$ 


\begin{abstract}
Determining multiscale, concurrent strain and deformation mechanisms in hierarchical biological materials is a crucial engineering goal, to understand structural optimization strategies in Nature. However, experimentally characterizing complex strain and displacement fields within a 3D hierarchical composite, in a multiscale full-field manner, is challenging. Here, we determined the in-situ strains at the macro-, mesoand molecular-levels in stomatopod cuticle simultaneously, by exploiting the anisotropy of the 3D fibre diffraction coupled with sample rotation. The results demonstrate the method, using the mineralized 3D $\alpha$-chitin fibre networks as strain sensors, can capture sub-micron deformation of a single lamella (mesoscale), can extract strain information of multiple constituents concurrently, and shows that $\alpha$-chitin fibre networks deform elastically while the surrounding matrix deforms plastically before systematic failure under compression. Further, the results demonstrate a molecular-level pre-strain gradient in chitin fibres, resulting from different mineralization degrees in the exo- and endo cuticle.
\end{abstract}

\title{
Keywords:
}

nanofibre networks, chitin-based biomaterials, nanoscale mechanics, in situ synchrotron wide-angle x-ray diffraction, fibrillar deformation, arthropod cuticle 
Helicoidal plywood (Bouligand) structures exhibit high damage-resistant performance in biological materials and bio-inspired composites, by forming energy-consuming twisting crack along rotating fibre directions. ${ }^{1-3}$ Structural and chemical gradients varying from the surface to the inner core of layered materials (bone, ${ }^{4}$ lobster exoskeleton, ${ }^{5}$ and Arapaima scale $^{6}$ ) is another ubiquitous concept from nature, to enhance the mechanics of natural materials relative to their homogeneous counterparts. ${ }^{7}$ Excellent mechanical strength and compressibility, particularly dynamic mechanical properties, are also essential factors that determine the impact resistance of materials. The stomatopod cuticle includes all those above-mentioned characteristics in one material while retaining a light weight, and is thus a biomimetic model for developing new materials in aerospace, automotive and armor applications. ${ }^{2,8-11}$ The cuticle of the club-shaped dactyl organs, used for inter- and intraspecific offense, can withstand repetitive load up to $1500 \mathrm{~N}$, while the defensive shells (telson) remain undamaged from forceful striking of the dactyls of other stomatopods during their ritualized fighting. ${ }^{12}$ The structural design of the cuticle has thus been intensively studied for investigating natural optimization strategies. Toughening mechanisms identified to date include the densification and collapsing of pore canals, ${ }^{13}$ reorientation and delamination between different fibre layers, ${ }^{6}$ and modulus mismatch between different lamellae, inducing crack deflection. ${ }^{11}$ However, a concurrent multiscale analysis of the deformation mechanisms throughout the entire hierarchical structure of cuticle during loading does not exist, with most analysis captured from pre- and post-loading (localized or global) 
of the tissue using combinations of electron microscopies and nanoindentation. ${ }^{14,15}$ Analogs to current structural motifs exist, but only in part. For example, at the microscale, a cuticle has a honeycomb -like structure, ${ }^{16}$ which is much more deformable - without losing strength ${ }^{16,17}$ - compared to man-made structural foams manufactured with carbon nanofiber ${ }^{18}$ and nanotubes, ${ }^{19}$ graphene ${ }^{20}$ and polymers. The honeycomb structure can only explain high deformability lateral to the cuticle plane but not for the normal direction of the cuticle. ${ }^{18}$ Further, the tissue is not a simple repeating plywood arrangement, but is modulated by a chemical and structural gradient at both the molecular and micrometer scale. The growth-induced chemical and structural gradients in biological composite materials (bone, ${ }^{21}$ tendon, ${ }^{22}$ etc.) are usually associated with the existence of intrinsic pre-strain and residual stress, which play significant roles in determining their macroscopic mechanical behavior; ${ }^{23}$ analogs may exist for cuticle as well.

Measurement of the multiscale deformation of nanocrystalline biocomposites like cuticle poses experimental challenges. At the molecular to nanometer scale, chitin fibrils ( 3-4 $\mathrm{nm}$ in diameter) self-assemble with proteins to form fibres $(\sim 20-100 \mathrm{~nm}$ in diameter). ${ }^{24}$ At the next hierarchical level, these fibres (referred to as fibres or nanofibres in the current work) aggregate inside a protein matrix, partially mineralized with calcium carbonate, to form planar arrays of fibres stacked in a twisted plywood manner to form lamellae at the microscale (along with a smaller component of fibres in the pore canals running perpendicular to the lamellae). X-ray transmission and CT 5 
measurements ${ }^{25,26}$ can potentially determine micron-level strains in a time-resolved manner, but do not resolve molecular-level structure. Diffraction-based 3D X-ray tomographic techniques like $\mathrm{x}$-ray diffraction contrast tomography ${ }^{27}$ differentialaperture $\mathrm{x}$-ray microscopy ${ }^{28}$ and dark field $\mathrm{x}$-ray microscopy ${ }^{29}$ can resolve nanoscale strains in macroscopic samples but require crystalline domains of the sizes of microns or larger, which is not the case for biological nanofibre composites. Coherent X-ray imaging methods $\mathrm{s}^{30}$ can measure strain at the molecular scale but have a restricted field of view of $\sim$ tens of $\mathrm{nm}$.

Here, we demonstrate the measurement of molecular-, meso, and macro-level strains in stomatopod cuticle by using a combination of micron-scale synchrotron X-ray diffraction, in situ compressive loading, and sample rotation to retrieve different slices of the $3 \mathrm{D}$ reciprocal space intensity, coupled with $2 \mathrm{D}$ scanning to resolve the strains at different meso-scale tissue locations. Our approach depends on molecular-level fibre symmetry (i.e. all crystallites have their $c$-axes aligned along the fibre axis, but the other two orthogonal directions have all possible orientations consistent with this constraint; see Methods/Experimental for further details). ${ }^{31-33}$ The technique exploits the 1D gradient in fibre orientation characteristic of the Bouligand plywood structure, which affects the diffraction intensity (Figure 1) of fibres oriented at different angles to the main axis. We use the fibre-angle dependent intensity to map the lamellar (meso) strains, combined with peak shifts of the on- and off-axis reflections to measure the molecularlevel strain. From these experiments, we uncover pre-strain gradients in cuticle across 6 
the tissue, measure spatially-resolved molecular strain and compare strains at different hierarchical levels developed in the exo- vs. endocuticle, and gain insights into how large, irreversible strains and energy absorption is transduced down to the fibre-level. 


\section{Results and Discussion}

\section{WAXD scanning and texture methodology for tracking single lamellae:}

The $\alpha$-chitin fibres form a plywood lamellar structure, further separated into exoand endocuticle, within the stomatopod tergite cuticle (Figure 1A), and peak shifts in the axial (002) and equatorial (110) reflections can be used as molecular-level strain sensors. As shown in Figure 1E-F, the intensity of these reflections depends on the angle the fibre makes with respect to the beam, which will vary periodically in a Bouligand type structure (Figure 1A and Figure 2F). In our sample-geometry, the stomatopod tergite is mounted with the surface at approximately right-angles to the incoming beam (Figure 1A), as can be seen from the 2D raster scan in Figure 2E. As a result, the fibre angle varies periodically along the vertical axis, as shown in idealized form in Figure 2F. As can be seen from Figure 1E-F, the WAXD pattern at each point along a 1D line scan (red circles in Figure 2E) will exhibit intensity variation in both (110) and (002) reflections as the fibre angle varies. The intensity variations can be predicted from a $3 \mathrm{D}$ diffraction model we have developed, ${ }^{31,32}$ which simulates the diffraction peaks as rings in reciprocal space (Figure 1E), as described in Methods/Experimental. In our model, the untilted fibre is as shown in Figure 1E. An initial rotation $\alpha=\pi / 2$ around the $q_{\mathrm{x}}$ direction (horizontal and at right angles to the X-ray direction in Figure 1E) is followed by a variable pitch rotation 
$\beta$ around the vertical axis, which will lead to different orientations as in Figure 1F and Figure 2F.
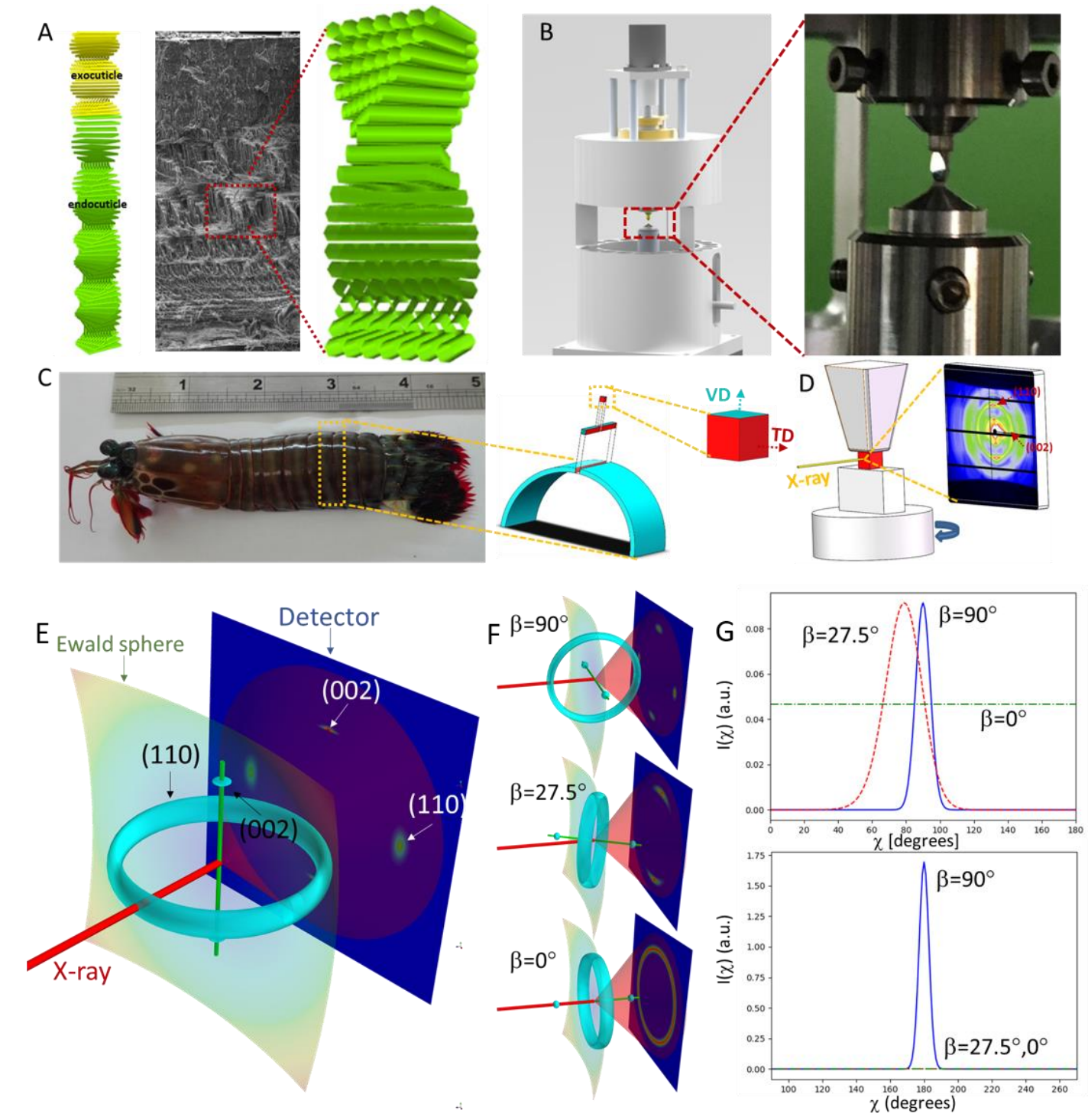

Figure 1: A): SEM image for the cross section of fractured stomatopod cuticle (middle) and a sketch of a tergite cuticle composed of multiple Bouligand-type lamellae (left), embedded in the matrix, consist of protein and minerals. The whole tergite cuticle is divided into exocuticle (yellow) and endocuticle (green) regions according to different fibre packing densities. The sketch on the right indicates a single lamella from the endocuticle region. $\boldsymbol{B}$ ): Sketch (left) and photograph (right) of the in-situ compression tester used for the synchrotron tests. C): Sketch showing how the specimen, used for synchrotron test, was collected from the stomatopod abdominal tergite cuticle. D): Schematic of the in-situ compression test on cuticle specimens by synchrotron WAXD, with an example $2 D$ WAXD pattern shown on the detector. E): The 9 
schematic showing $3 D$ intensity distribution of the (002) and (110) diffraction signals from a chitin fibre in the reciprocal space. F): The (110) and (002) pattern change as the fibre orientation changes. Here, $\beta$ refers to a tilt around the vertical axis. A tilt away from the vertical axis $(\alpha)$ is taken as $\pi / 2$ exactly in this case. $G$ ): The $I(\chi)$ curve of (110) (top) and (002) (bottom) reflections acquired from different fibre orientations for an idealized Bouligand structure.

To demonstrate how the 3D texture of the (110) reflection can help identify the periodic lamellar structure, the sample was scanned in 1D (along vertical axis; red circles in Figure 2E) coupled with a rotation around the vertical axis. The experimental WAXD patterns are shown in Figure 2B, and the integrated (110) intensity via a $z-\varphi$ plot ( $\varphi$ the rotation angle) in Figure 2C. A clear periodic diagonal banding is observed in the $z-\varphi$ plot, with the period being smaller in the exo- $v s$. endocuticle. To show that this periodic diagonal banding arises predominantly from the fibre angle, we simulate the predicted (110) intensity for the Bouligand twist pattern in Figure 2F using our 3D diffraction model, with the results shown in Figure 2G. A clear qualitative similarity is observed between the experimental and predicted diagonal banding. The (110) diffraction intensity can therefore be linked to the fibre angle (See Supplementary Information, Figure S7 for a plot of (110) intensity $v s$. fibre angle) and by profiling in z-direction, can determine the boundaries of individual lamellae in exo- and endocuticle. The thicknesses of the lamellae in exo- and endocuticle before loading, deduced from the vertical height of those strips, are 10-15 $\mu \mathrm{m}$ (exo-) and 20-50 $\mu \mathrm{m}$ (endo-), in good accordance with values collected from SEM results. ${ }^{32}$ 
It is noted that the observed (110) peaks in the 2D WAXD patterns in Figure 2B are not separated by exactly $180^{\circ}$, due to the Ewald-sphere curvature effect when the fibres are slightly tilted away from the idealized Bouligand structure shown in Figure 2F. This effect is related to the Fraser correction for fibre diffraction analyzed previously $;{ }^{34}$ in terms of our model it corresponds to the fibre tilt angles $\alpha, \beta$ deviating slightly from $\pi / 2$. This small additional tilt is disregarded in our simulation in Figure 2G. While for a general 3D fibre orientation distribution, simple rotation around a single axis would not suffice for a full reconstruction, and advanced 3D reconstruction methods (e.g. as recently reported for WAXD-tensor tomography on bone $^{35}$ ) would be needed, the single axis rotation works for the specific case considered here - a 1D variation in fibre angle due to the Bouligand structure, and a need to determine meso- and molecular-level strains.

A second effect which has been neglected is the change in scattering volume when the rectangular $\left(0.5 \times 0.4 \mathrm{~mm}^{2}\right)$ square sample is rotated around the vertical axis, leading to greater diffraction intensity due to increased path-length, counterbalanced by increased absorption, when the beam is going along the diagonals of the sample when viewed from the vertical direction (Supplementary Information, Figure S4). Further, two vertical bands $\left(\sim 30^{\circ}\right.$ and $\left.\sim 150^{\circ}\right)$ occur when the diffraction signal is blocked by one of the three spacer-pillars connecting the top and bottom parts of the compression tester (Figure 1B). 


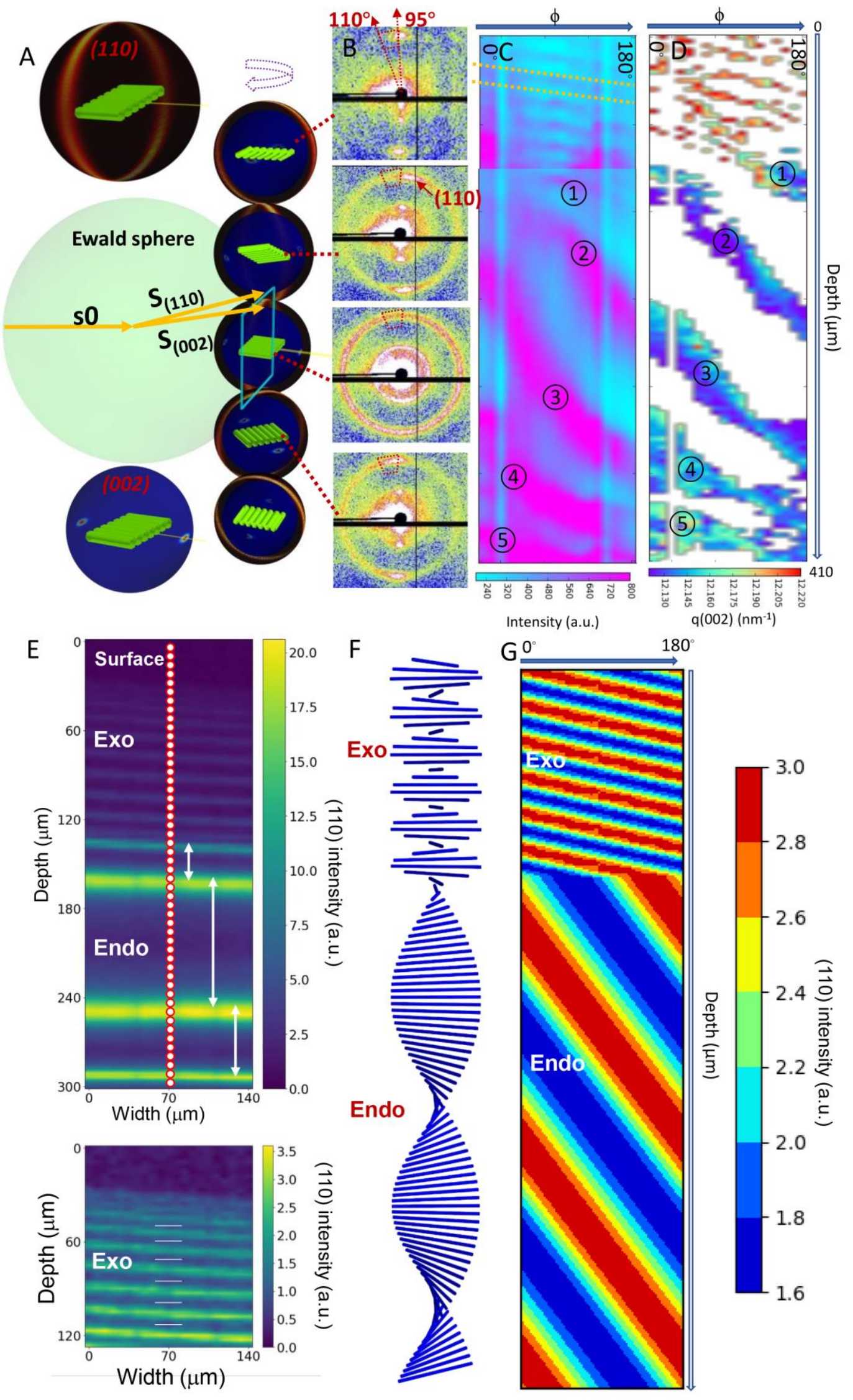


Figure 2. Probing the deformation mechanisms of mesoscale lamella and sublamellae of varied orientations within stomatopod tergite cuticle through tomographic alike WAXD scans during in-situ compression. A): Sketch of the intersection geometry between $3 D$ reciprocal (110), (002) spheres and Ewald sphere; $\boldsymbol{B})$ : The diffraction patterns collected from a raster scan across a single lamella in the synchrotron experiments showing the variation of the (110) reflection pattern. The red dash rectangles on the diffraction patterns indicate the azimuthal region used for calculating the intensity of (110) reflections; C): The (110) intensity map acquired from the raster scan across the cuticle with the rotation of sample. A tilted strip (indicated by yellow dash lines) on the intensity map indicates a plywood lamella. Since the intensities in the exocuticle region were significantly lower than those in the endocuticle region, the values in the exocuticle region shown in Figure $2 C$ were multiplied by 3 for display purpose. Note that the whole cuticle thickness was not scanned during the pseudo-tomographic scans; the encircled numbers (1) (5) indicate five separate lamellae in the endocuticle region. D): The q(002) value map acquired from the raster scan across the cuticle with the rotation of sample. The q(002) values in the exocuticle region were higher than the ones in the endocuticle region. E): The (110) reflection intensity map acquired from a $2 D$ WAXD scan on the tergite cuticle. $\boldsymbol{F})$ : The schematic of tergite cuticle with plywood structure and different lamella thickness in the exo and endocuticle. G): The modelling results of (110) intensity map acquired from the raster scan across the cuticle with the rotation of sample shown in $\boldsymbol{F}$

\section{Discovery of a pre-strain spatial gradient at the nanofibre level in chitin:}

Figure 2D shows a map of the reciprocal wavevector corresponding to the $\mathrm{D}(002)$ lattice spacing as a function of $\mathrm{z}$ - (vertical axis) at different rotation angles. The intensity of the axial (002) WAXD reflections from different sublamellae exhibits a texture effect, rotating into the Ewald diffraction condition when fibres are (approximately) normal to the beam (Figure 1F and Figure 2A). As a result, on scanning across a lamella, the value for the (002) peak is obtainable only when the fibre is rotated into diffraction condition, accounting for the gaps (white spaces) in Figure 2D. In Figure 2D the most striking observation is that the wavevector corresponding to the (002) lattice spacing $\mathrm{D}_{(002)}$ in the exocuticle is clearly larger compared to the endocuticle (reddish coloration in exocuticle vs. blue- 
green in endocuticle), i.e. $\mathrm{D}(002)$ is smaller in the exo-cuticle versus the endo-cuticle. The average $\mathrm{D}_{(002)}$ increases by $\sim 0.37 \%(\sim 5.155 \AA$ to $\sim 5.174 \AA)$ from exocuticle to endocuticle region. This finding demonstrates the existence of a pre-strain gradient in the chitin nanofibre network across the tergite exoskeletal thickness. Chitin fibres in the exocuticle are pre-compressed by about $0.37 \%$ relative to those in the endocuticle, which translates to a substantial stress difference of $0.56 \mathrm{GPa}$, given the relatively high Young's modulus of chitin fibres (150 GPa) obtained from ab-initio simulations and experiment. ${ }^{36}$ The difference is statistically significant $(p<0.001)$ between the exo- and endocuticle - in Supplementary Information, Figure S2-D shows the results from 3 cuticle samples scanned using z-axis scanning coupled with $\phi$-rotation, with the (002) D-period significantly lower in exo- $v s$. endocuticle in each case.

We note that the difference is not likely to arise due potential differential drying between the exo- and endocuticle over the course of the scan. This is evidenced by similar measurements we carried out at beamline I22, Diamond Light Source (DLS) where the sample was kept in a fluid chamber. The last pair of averaged values in Figure S2-D shows that a similar decrease of (002) D-period is obtained in the exocuticle $v s$. the endocuticle for the measurements carried out at DLS. 

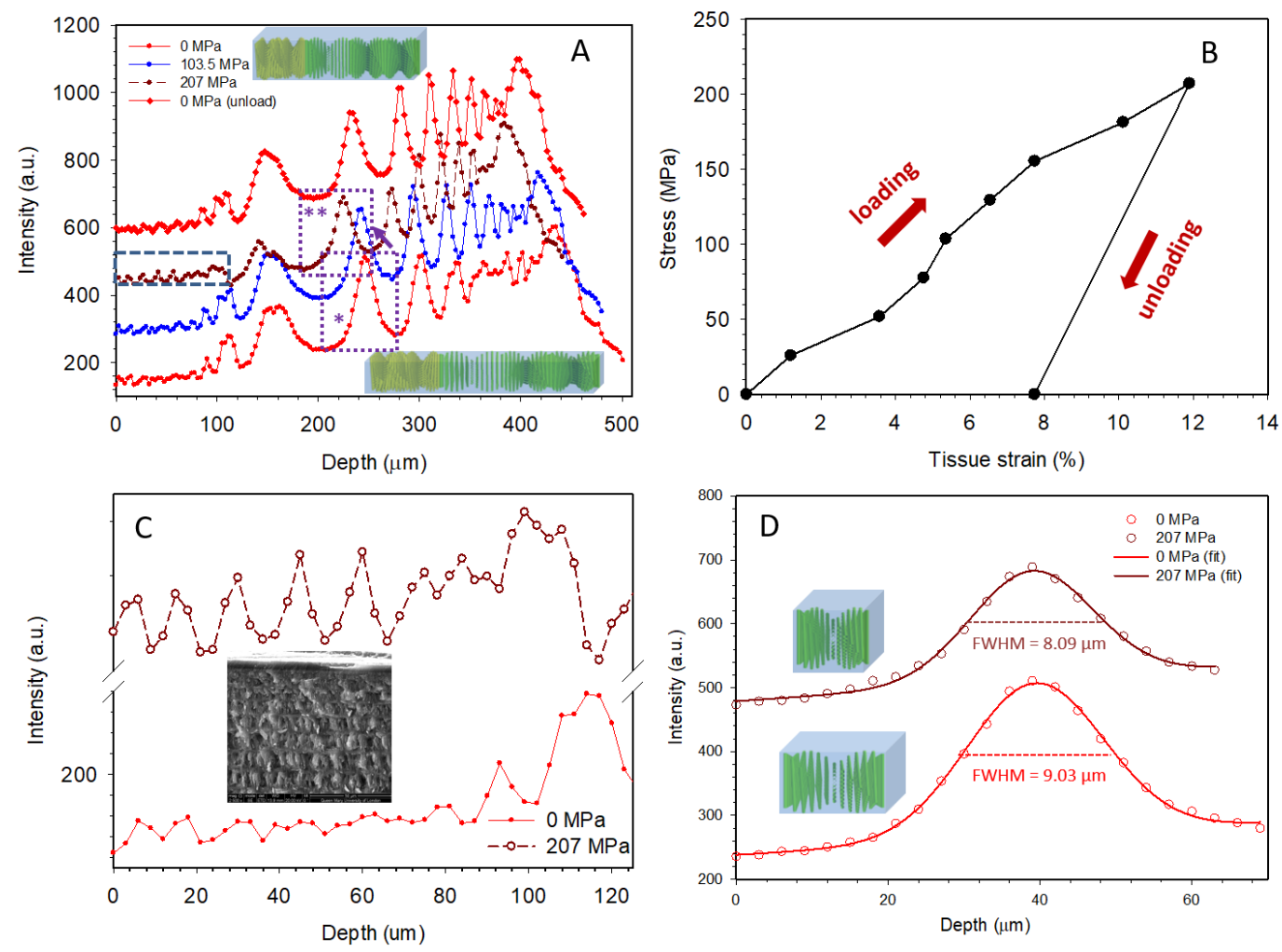

Figure $3 \mathrm{~A}$ ): The (110) diffraction intensity distribution across the exo- and endo-cuticle at different loading stages. Curves are vertically shifted for clarity. Different lamellae across the depth of the tergite cuticle can be identified from the periodic intensity change of (110) diffraction. B): The tissue stress versus applied compression load, showing residual strain on unloading. $\boldsymbol{C}$ ): An enlarged plot from the selected region (blue rectangle) shown in $\boldsymbol{A}$ demonstrates the thin lamellae in exocuticle region can be separated using a $3 \mu \mathrm{m}$ scanning step, and the change with load. D): The (110) diffraction intensity distributions of the second lamella from the endocuticle region were selected from the plots in $\boldsymbol{A}$ (indicated by the purple dotted rectangles); the decreasing FWHM of the intensity curve b when under 207 MPa compression stress compared to unloaded state indicates that the deformation of a single lamellae along the loading axis can be quantified.

Concurrent strain-determination at macro-, meso-, and molecular-scales:

From the lamellar structure (Figure 2C) and the D-period (Figure 2D), it is possible to calculate the meso (lamellar-level) and nano/molecular (fibre-level) displacements and strains with sub-lamellar level resolution, jointly with 
macroscopic (tissue-level) strains. A full (albeit time-consuming) reconstruction will require $1 \mathrm{D}$ - or $2 \mathrm{D}$ scanning, coupled with single-axis $\phi$-rotation for each sample. However, if only lamellar-level structure/strains are needed, 1D- or 2D-scans of the cuticle without $\phi$-rotation are sufficient, as can be seen by taking a vertical lineprofile down the z-axis in Figure 2C. The speed of such measurements ( $\sim$ minutes) is much less than the full multi-axis scan ( hour). We will first show lamellar-level displacements from in-situ compression of cuticle along the vertical axis (abbreviated scans without $\phi$-rotation), and then the molecular-level strains (full scans including $\phi$-rotation).

Figure 3A shows the periodic oscillation of (110) diffraction intensity, arising from local fibre orientation in each sub-lamella, and the change in this periodic pattern on loading stepwise to $12 \%$ macroscopic strain (in increments of $25 \mathrm{MPa}$; see Methods/Experimental). WAXD line scans from two selected load levels ( 100 and $200 \mathrm{MPa}$ ) are shown, along with the initial and unloaded states. The characteristics of the stress-strain curves are reproducible, with Figure S4 in Supplementary Information showing stress-strain curves from three samples. On unloading to zero stress, the macroscopic strain is not fully recoverable on unloading (residual strain of $\sim 7.7 \%$ ). Due to the thinner lamellae in exocuticle compared to endocuticle ${ }^{5}$, zoomed-in graphs (Fig 3C-D) can be used to analyze the strains in the exo- and endocuticle more clearly. By comparing full width at half-maximum (FWHM) value of the (110) intensity peak in the second lamella in endocuticle before 16 
and under loading (Figure 3D), an $\sim 11 \%$ strain at $207 \mathrm{MPa}$ compression stress is observed.

As strain is a first-order perturbation on the resolved intensity curve, with the current spatial resolution of $3 \mu \mathrm{m}$ (scan step size), we can resolve microscale strains only for thicker lamellae (thickness $\sim 30 \mu \mathrm{m}$ in endocuticle). However, the resolution limit can be progressively increased by decreasing raster step-size and beam diameter, enabling deformation of even sub-micron structures (limited by radiation sensitivity of the material).
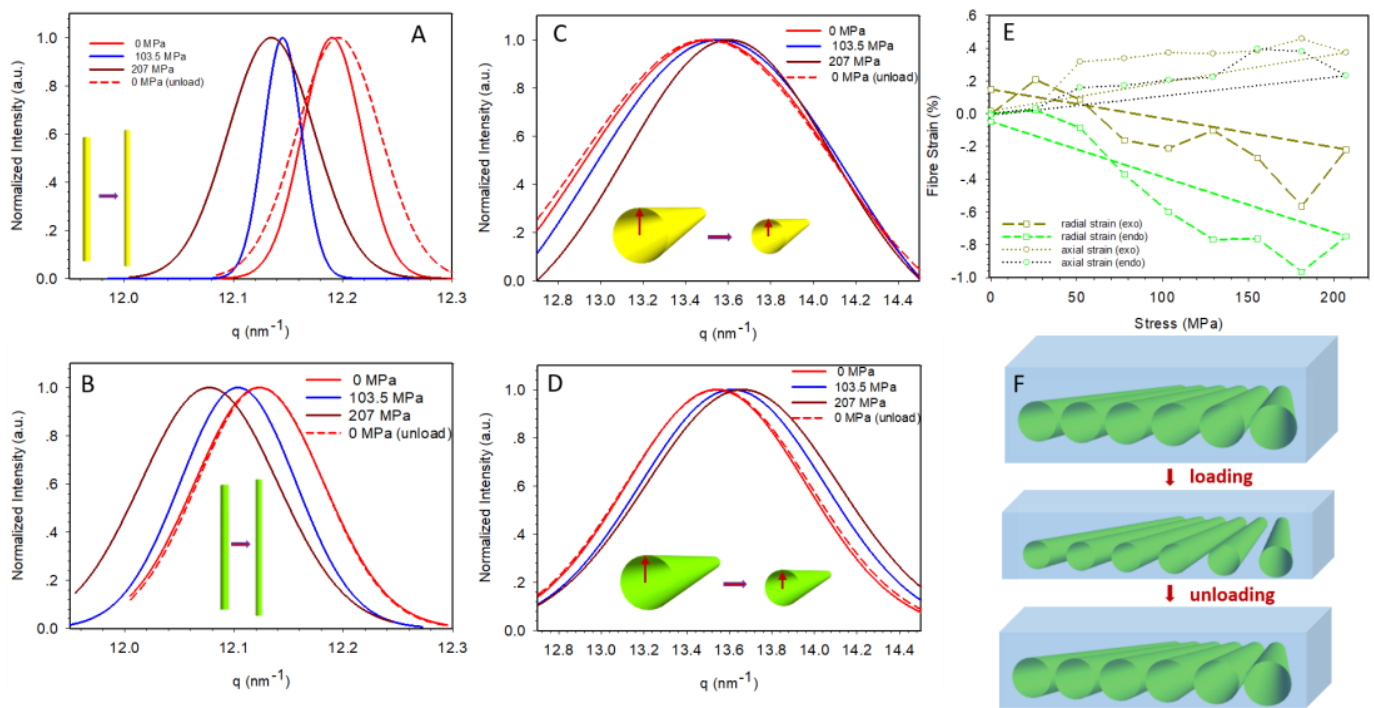

Figure 4. A-B): The (002) chitin reflection peaks collected from exocuticle $(\boldsymbol{A})$ and endocuticle $(\boldsymbol{B})$ in the I(q) plots shift negatively under increasing compression loads. $\boldsymbol{C}, \boldsymbol{D})$ : The (110) chitin reflection peaks collected from exocuticle $(\boldsymbol{C})$ and endocuticle $(\boldsymbol{D})$ in the $I(q)$ plots shift positively under different compression loads. $\boldsymbol{E})$ : The radial and axial strain of the fibres from exo-and endocuticle were plotted versus different loading levels, where the radial strain values were obtained by tracking the shifting of the (110) diffraction peak of the diffraction pattern, while the axial strain values were acquired by tracking the shifting of the (002) diffraction peak of the diffraction pattern. $\boldsymbol{F})$ : A schematic showing that the deformation mechanism of the fibres within the matrix. 

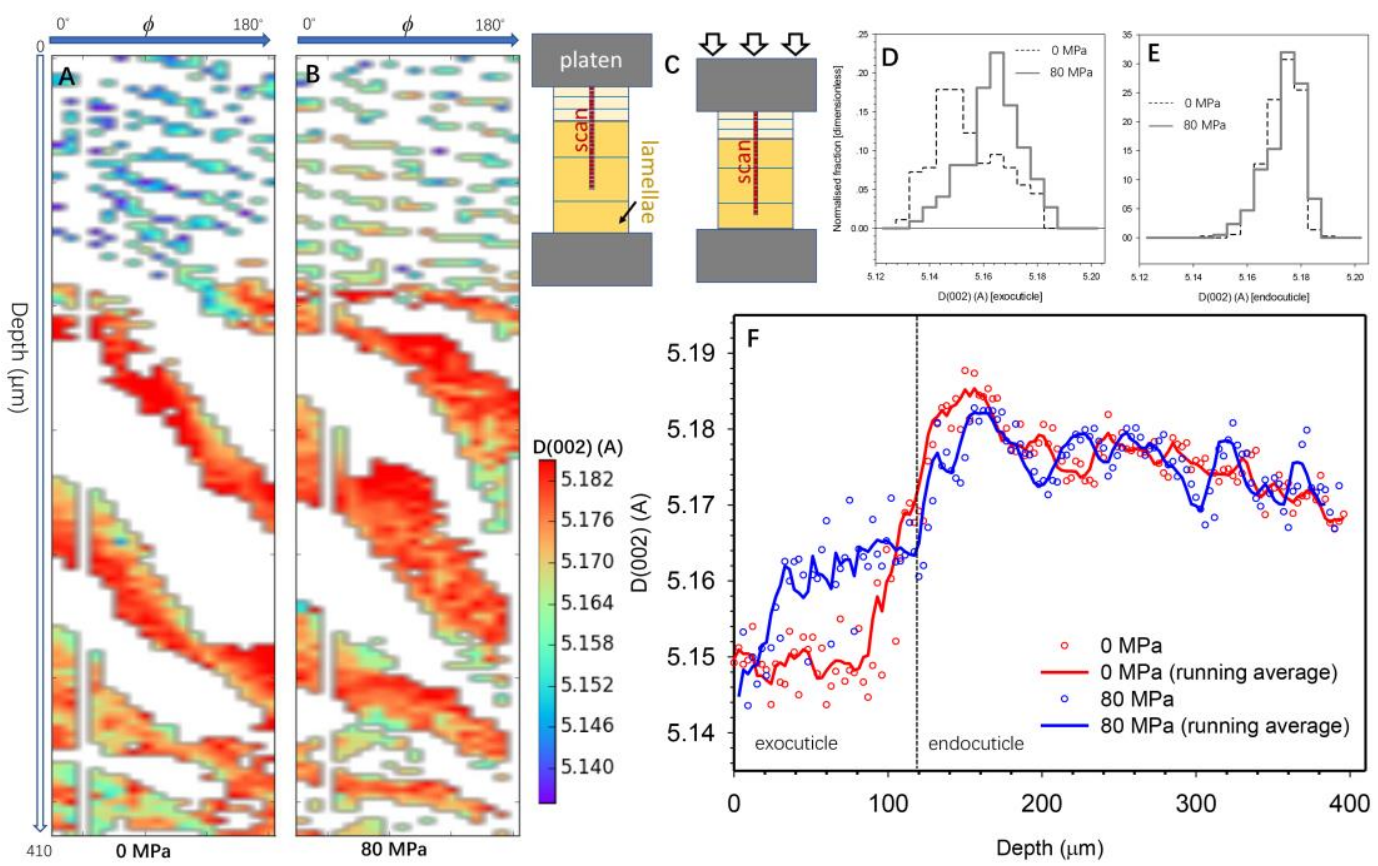

Figure 5. $\boldsymbol{A}-\boldsymbol{B})$ : The $D(002)$ value map acquired from the raster scan across the cuticle with the rotation of sample before and under 80 MPa compression loading. C): A schematic showing less lamellae were scanned as the sample was contracted under compression. D-E): showing the $D(002)$ value distribution in exo- and endocuticle before and under compression loading. $\boldsymbol{F})$ : The $D(002)$ value changes across the exo-and endocuticle when under compression loading. The data shown in $D, E, F$ were acquired from $A, B$.

The molecular-level strains are obtained from percentage shifts in the (002) and (110) peak positions: the axial fibre strain is determined from the (002) peak shift, and the transverse fibre strain from the equatorial (110) peak shift. Considering first the average molecular strain in exo- versus endocuticle, Figure 4A-B (exo-) and C-D (endo-) show peak shifts of the order of $0.4 \% / 1.0 \%(002) /(110)$ in the exocuticle and $\sim 0.4 \% / 0.5 \%$ in the endocuticle. As expected, vertical compression on the horizontally oriented fibres leads to axially elongated and radially compressed fibres 
(Figure 4F). The axial elongation in the fibres $(\sim 0.4-0.5 \%)$ is similar in magnitude to the radial compression in the exocuticle. However, in the endocuticle, it is about half $(\sim 0.5 \%$ vs. $1.0 \%)$, indicating that the fibres in the endocuticle bear a larger compressive strain fraction. Possible nanoscale matrix/fibre interfacial damage, characterized by lower stresses transferred to fibres from the interfibrillar matrix, is indicated by clear drops in fibre strain on increasing stress from to $181 \mathrm{MPa}$ to 207MPa.

Two notable contrasts between the microscale and nanoscale deformation behavior are seen when comparing Figures 3 and 4. First, the fibre strains are more than an order of magnitude smaller $(<1 \%)$ than the tissue/macro strains $(\sim 10 \%)$. Second, while at the tissue level, substantial residual strain remains on unloading, at the nanoscale, chitin fibres lower stresses transferred to fibres spring back to their original shape on removal of applied compression loads. These results suggest that plastic deformation and dissipation of the impact energy occurs mainly in the interfibrillar matrix, whilst the 3D fibre networks mostly undergo elastic deformation, avoiding damage by detaching from the matrix at high stress.

Progressing to spatially resolved maps of molecular strain, Figure 5A-B shows the change in (002) D-spacing on combined z- $\varphi$ scanning upon application of 80 MPa stress. From both the 2D color plot (Figure 5A-B) and more clearly from the line-plot (Figure 5F, averaging D-spacing values at the same z-coordinate), it is seen 
that there is a clear stress-induced increase in axial fibre D-spacing in the exo-cuticle on average. In the endocuticle, there is less of a similar lateral shift. This is corroborated by D-period histograms in each region (Figure 5C-D), which show little shift in the distribution in the case of the endocuticle (Figure 5D). This finding implies that chitin fibres initially develop significant strain in-situ on compressive loading mainly in the exocuticle, while they strain less in the endocuticle. Factors enabling this difference may include i) the compressive pre-strain in the exocuticle (Figure 2D), giving the fibres some slack to stretch initially, absent in the endocuticle and ii) the lower degree of interfibrillar mineralization in the endocuticle, enabling greater compressibility of the interfibrillar matrix, and a lowered strain fraction as a result. It is possible that the increased radial compaction (Figure 4E) in the endocuticle may also play a role.

Closer inspection of Figure 5F in the loaded case shows that the axial $\mathrm{D}(002)$ spacing exhibits oscillations between lamellae in the exocuticle. The width of these oscillations is same as the lamellar size in the exocuticle, suggesting that differently angled fibres (Figure 2F) in the exocuticle may have variable pre-strain levels. A similar effect is seen in the endocuticle, although the period of oscillation is not as constant, possibly linked to the variable endocuticle lamellar width.

From the above, the nanofibre network in cuticle behaves like a well-connected 3D polymeric foam, retaining the structural integrity to withstand external impact 
loading, whilst the highly mineralized matrix acts like concrete surrounding iron girders (analog to fibre networks in cuticle) in civil construction. ${ }^{37}$ The highly mineralized matrix system enables the cuticle to have excellent dynamic mechanical performance by dissipating more energy through plastic deformation. However, without the structural integrity provided by the nanofibre network, which prevents cracks from propagating straight through the tissue, the cuticle would break in a brittle manner. Besides achieving better mechanical stability, the design also gains biological benefit for reducing the metabolic costs of repair and renewal of a complex composite. It is less metabolically expensive to deposit new matrix (of amorphous minerals) rather than rebuilding a new 3D hierarchical fibre network.

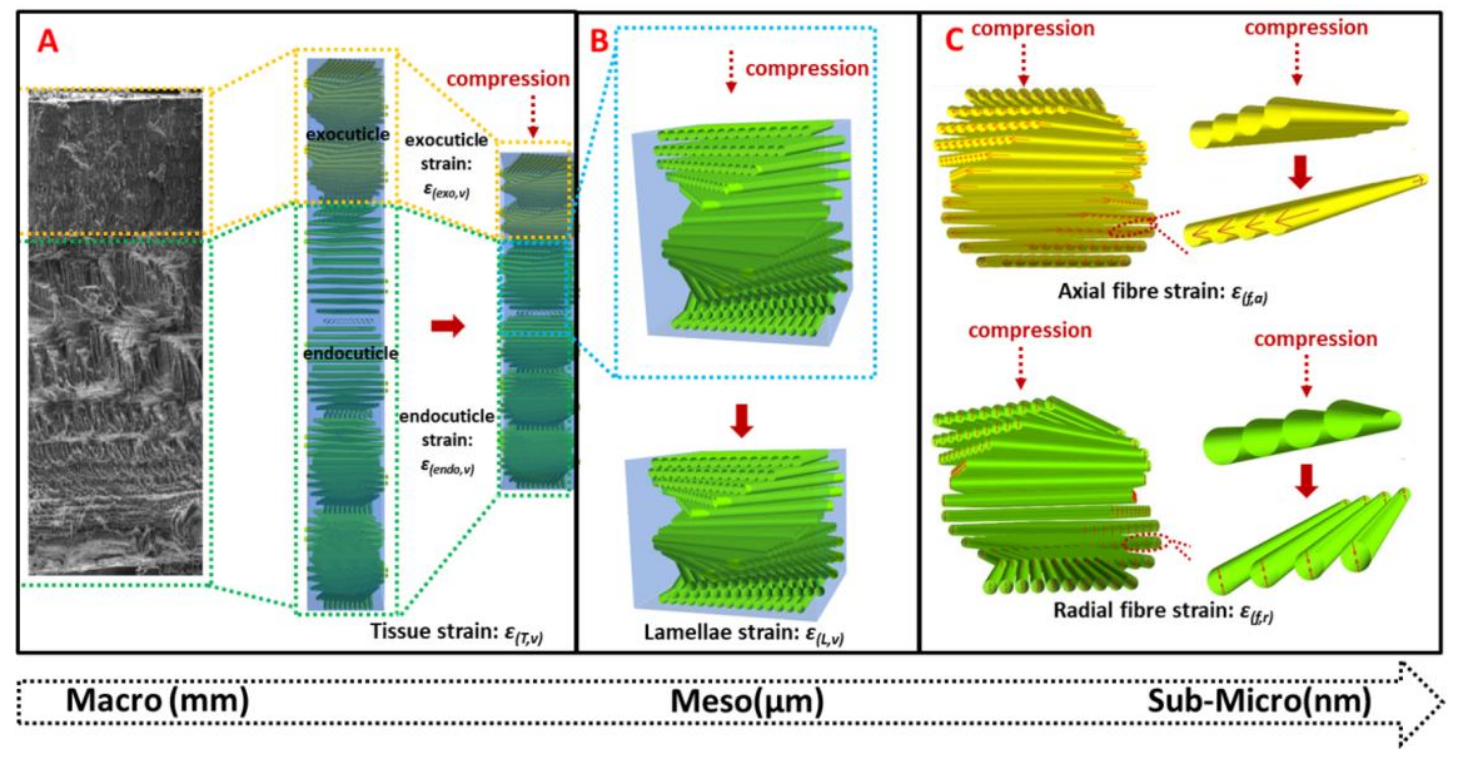

Figure 6. Probing the multi-scale deformation mechanism of the stomatopod tergite cuticle in a single in-situ compression test. A): In the macroscale, the deformation of the whole cuticle, or the exocuticle and endocuticle as a whole can be determined by the periodic oscillations of the (110) diffraction intensity curve or the differences in the transmission curves; $\boldsymbol{B})$ : In the mesoscale, a single plywood lamellae can be identified from oscillating (110) diffraction intensity curves and its deformation can be detected using fine raster scans. $\boldsymbol{C}$ ): In the nanoscale, 
the deformation of a sub-lamellae can be examined by checking the peak shift of (002) and (110) reflection from the chitin fibres.

To summarize our experimental results for discussion, our main findings on the small-scale mechanics of cuticle include: a) a molecular-level pre-strain gradient in the chitin fibres between the exo- and endocuticle (Figure 2), with the chitin fibres pre-compressed in the higher mineralized exocuticle; b) a decrease in strain-levels by over a factor of 10 when going from the tissue to the molecular-scale (in the chitin) (Figures 3-4); c) higher degree of radial compaction (viz. axial extension) of chitin fibres in the endocuticle $v s$. the exocuticle (Figure 4); d) under loading, a different response of the chitin fibres in the exocuticle (Figure 5) compared to the endocuticle; e) evidence that the chitin nanofibres act as an essentially elastic spring-like network even under irreversible macroscopic deformation, as shown by molecular-level strains returning to zero while residual strain of $\sim 8 \%$ remains at the macroscale (Figures 3-4). Such a system strikes a balance between structural stability, high compressibility $(\geq 15 \%)$ and energy dissipation efficiency at low metabolic costs for tissue repair and renewal. This design concept from nature may be replicated by advanced three-dimensional printing techniques ${ }^{38}$ to develop bioinspired high mechanical performance composites while retaining low weight for structural materials in the aerospace and defense industry, ${ }^{8}$ as well as piezoresistive sensors and supercapacitors. ${ }^{39}$ 
A conceptual sketch of these multiscale mechanisms is shown in Figure 6. Indeed, using the elastic nature of the chitin fibres, one can potentially use the multiaxial strain in the chitin fibres (axial (002) and transverse (110)) as local stress sensors to track the deformation of the interfibrillar matrix, mainly composed of amorphous minerals and proteins. The approach put forward here is potentially generalizable to other hierarchical biological or synthetic composites with fibre symmetry. The results extend our knowledge of the mechanics of the tough and impact resistant cuticle. Prior work has extensively characterized the macroscopic and microscopic structure $^{11,14,40,41}$ but the knowledge of the in-situ mechanics and strains in the different nanoscale compartments has been less studied. This scale is challenging to probe both because of the small length-scale (which can be addressed using probes such as micro- and nano-WAXD) as well as due to the challenges of interpretation of the complex anisotropic 3D diffraction signal. Recent work on other biological composites using small X-ray beams and 3D reconstruction include nano-WAXD diffraction tomography to probe structural and compositional variations over macro(tissue-level) scales in other biological composites like bone, ${ }^{42}$ and 3D nanostructural variations in bone mineral has recently been uncovered using WAXD tensor-tomography ${ }^{35}$ and SAXS tensor tomography ${ }^{43}$ approaches. Alternative, energy-dispersive X-ray scattering with specialized detectors can uncover 3D reciprocal space structure in chitin and cellulose systems. ${ }^{35}$ However, these prior 
works do not incorporate, to the best of our knowledge, in-situ mechanical probes of the nanostructure.

Strains in the mineral phase at the molecular level in biological composites have been noted before, e.g. in bone ${ }^{44}$ using high-energy X-ray diffraction combined with insitu compression. More recently the role of hydration and mineralization affecting collagen pre-strain and D-spacing in bone, cartilage and tendon have been brought out via in-situ hydration and mechanical studies. ${ }^{45-48}$ Our recent work ${ }^{49}$ has revealed that (taking cuticle as a whole), the axial (002) lattice spacing of chitin increased upon demineralization, followed by a decrease on partial deproteinization. It is also known that the mineralization of exocuticle is larger than in endocuticle. ${ }^{50}$ Taking these two findings together, it is possible that the lower (002) D-spacing in the exocuticle is linked to its greater degree of mineralization. However, in the absence of knowing the zero-stress reference state of the chitin nanofibre, it is not clear whether to interpret the reduced (002) D-spacing in the exocuticle as a compressive pre-strain, or a loss of pre-existing tensile pre-strain. We note also that the axial width for the (002) peak in the exocuticle (Figure 4A) is less than the axial width in the endocuticle (Figure 4B), supporting the idea that the change is due to a loss of tensile pre-strain: the application of tensile pre-strain (in the endocuticle) may cause chitin fibres to deform heterogeneously, leading to a larger peak width in the endo-versus the exocuticle, as well as a larger $\mathrm{D}(002)$ value. 
This reduction in tensile pre-strain (or increase of compressive pre-strain) in the unloaded exocuticle may provide some slack for axial extension of the fibres (e.g. in the loading geometry in our work, where a compressive load on the tissue surface results in axial elongation of the perpendicular oriented fibres). Possibly, this is the reason for the clear increase in D-period at low stress levels ( 80 MPa) seen in the exocuticle (Figure 5F) viz. the endocuticle. Subsequently, once this slack is removed, it can be speculated that interfacial failure or matrix-fibre debonding may occur at larger strains, evidenced by a broadening of the (002) peak in the exocuticle at higher loads $(\sim 170-210 \mathrm{MPa})$.

The hierarchical pattern of strains developed in the cuticle bears similarities to the multiscale strain-values observed in bone, ${ }^{51,52}$ and is part of the mechanical design of such fibre composites ${ }^{53}$ where interfibrillar shear between fibres at multiple scales accounts for the remaining strain. The strain-fraction of $\sim 0.1$ (fibre/macro) is smaller than that in bone fibres $(\sim 0.4-0.5)$ or mineral $(\sim 0.2)^{51}$. Broadening of the axial peak (Figure 4) is interpreted as due to interfacial damage, as partial debonding between the fibre and matrix will lower the strain on the fibre (and overload neighboring fibres), leading to a heterogeneous distribution of (002) D-periods and peak broadening.

Our method as presented can be adapted to other biological composites with fibre symmetry, but limitations of the current work need to be noted. Most importantly, 
we can draw conclusions about fibre and lamellar deformation because our sample geometry is relatively simple - a vertical stack of fibres oriented in the horizontal plane at different angles, i.e. a $1 \mathrm{D}$ variation in pitch angle for a $3 \mathrm{D}$ structure. As shown in Figures 1-2, in this case, intensity and fibre angle correlate simply. An incremental improvement would be to fit the diffraction model with nonzero $\alpha$ (and variable Bouligand pitch $\beta$ ) to the data, to account for slightly tilted lamellae relative to the beam. More complex fibre geometries (e.g. with multiple lamellae orientations in the beam) would require WAXD-tensor tomography-type measurements or other advanced techniques. ${ }^{35}$ Combining 1D- (or 2D-) scans with $\phi$-rotations for all samples and load-levels would be ideal (rather than only a small subset) but was not feasible due to both the long scan duration (tens of hours), and possible X-ray induced structural deterioration. Further, fibre rotation (in $\beta$ ) during loading may bring fibres into the Ewald diffraction condition, changing the (110) intensity (e.g. between the curves in Figure 3A). Here, we note that our sample-geometry (vertical stack of 2D lamellae) and loading-mode (vertical compressive stress) leads to much less interfibrillar shear than other modes like axial torsion or lateral shear. Hence, we expect this effect is playing less of a role, but further refinements would include fitting the $I(\chi)$ curve with variable $\beta$ before and after loading, to model the rotation around the axis. Lastly, microscale inelastic fracture mechanisms like crack propagation or deformation of the honeycomb structure are not analyzable by the 
method and need independent measurements by, for example, tomographic techniques. $^{25}$

\section{Conclusion}

In summary, we have shown how the multiple structural levels in stomatopod cuticle, a biological impact-resistant nanocomposite, responds at the nano-, micro and macroscopic scale on in-situ loading, by using a combination of scanning synchrotron X-ray diffraction and 3D reciprocal space modelling. Beyond the work presented here, the approaches for measuring strains from multiple length scales may be well suited to study other composite materials, of which the functional performance were governed by the structural and mechanical arrangements of their selected nanoscale crystalline components (such as metallic nanofibers, carbon nanotubes, ${ }^{54,55}$ and polymeric fibres. ${ }^{56}$

\section{Methods/Experimental}

\section{Sample preparation}

Mantis shrimp (Odontodactylus scyllarus) from the tropical Indo-Pacific were obtained from a commercial supplier (Tropical Marine Centre, London) and stored at $-20{ }^{\circ} \mathrm{C}$ till used for sample preparation. Samples were then acquired from the central region of the abdomen tergite cuticle using the same preparation method shown in our previous paper. Typical dimensions of specimens were $\sim 0.5 \mathrm{~mm}$ 
(thickness) $\times 0.35 \mathrm{~mm}$ (width) $\times 0.6 \mathrm{~mm}$ (length) with the width of the sample smaller or match the width of the loading tip $(0.4 \mathrm{~mm})$ the compression tester (Figure 1C).

In-situ compression testing

The in-situ compressive tests were performed using an in-house developed compression tester (Figure 1B). To record the force during the compression tests, a load cell was equipped in the compression tester with a maximum capacity of $50 \mathrm{~N}$. The control of the compressive load cell was integrated into the beamline control system. Prior to the compressive loading, a few drops of water were applied on the sample to wet it. During testing the sample was exposed to ambient air without applying any moistening.

A monochromatic $x$-ray beam $(17 \mathrm{keV})$ with a size of $1.9 \mu \mathrm{m}$ (horizontal) $\times 2.7 \mu \mathrm{m}$ (vertical) was used to raster scan the sample with a step size of $3 \mu \mathrm{m}$ and exposure time of $0.2 \mathrm{~s}$ per point. The WAXD images were collected using a photon counting Eiger 4M detector with a sample to detector distance of $169 \mathrm{~mm}$ (Figure 1D). The X-ray attenuation data was collected simultaneously as the recording of the diffraction image using a combination of ion chamber (upstream of the sample) and beam stop diode (downstream of the sample). The corresponding attenuation values were then used for the normalization of the diffraction signal. 
For the z- $\phi$ scan WAXD tests, the WAXD raster scans were recorded for 19 different rotation angles $\phi=0^{\circ}, 10^{\circ}, 20^{\circ}, \ldots 170^{\circ}, 180^{\circ}$ of the pre-aligned rotation axis. For the single-orientation WAXD test, a 1D WAXD line scan was performed on the sample as the force was increased to different levels. The force was kept held at each value with a $3 \%$ tolerance to avoid stress relaxation during the raster WAXD scans. Prior work on lobster tergite cuticle (a different species) ${ }^{13}$ shows a maximum stress $\sim 150 \mathrm{MPa}$, and lab-tests on stomatopod tergite samples prepared as above showed a maximum stress at $\sim 200 \mathrm{MPa}$. We hence took measurements at $\sim 25 \mathrm{MPa}$ steps from $0 \mathrm{MPa}$ up to $\sim 200 \mathrm{MPa}$. The stress was then unloaded, and a line-scan was carried out to examine the recovery process.

\section{WAXD data reduction}

The azimuthal integration process for the WAXD patterns collected in the raster scans was performed using pyFAI ${ }^{57}$ For tracking the periodic changes of the $(110)$ reflection to acquire the tissue strain, an azimuthal range between $95^{\circ}$ to $110^{\circ}$ was used for the integration (Figure 2B), as the diffraction intensity in the sector just offaxis to the vertical $\left(90^{\circ}\right)$ direction is found to give the largest contrast (change) with fibre rotation around the horizontal ( $\beta$-rotation as described in the Analysis subsection following). For probing the peak shifts of the (110) and (002) reflection to obtain the radial and axial fibre strain, an azimuthal range between $-10^{\circ}$ to $10^{\circ}$ or between $80^{\circ}$ to $100^{\circ}$ was used for the integration of (002) of (110) respectively. 
Following the azimuthal integration, the I(q) curves were further fitted using the same protocol described by us previously to track the shifts of $\mathrm{q}_{(002)}$ and $\mathrm{q}_{(110)}$ values. $^{31}$

Data analysis and modelling of the $3 D$ diffraction signal

For nanoscale composites containing inclusions with molecular-level fibresymmetry, the diffraction/scattering intensity points in reciprocal space transform into rings (Figure 1E) ${ }^{32,43,58}$. The intersection of the reciprocal space ring with the Ewald sphere (corresponding to the measured WAXD signal) changes when the angle of the fibre to the incoming beam changes. In our prior work ${ }^{31,32}$, we have derived analytical expressions for the measured 2D WAXD diffraction intensity as a function of both reciprocal wave vector $q$ and the $3 \mathrm{D}$ tilt of the fibre angle $(\alpha, \beta)$ with respect to the vertical axis, for the axial $(002)^{31}$ and equatorial/transverse $(110)^{32}$ reflections. While the (lengthy) expressions are reproduced in the Supplementary Information for reference, we note here that the case of the fibres stacked vertically (Figure 2E) with a Bouligand-type twisting of the fibre arrangement around the $z$ axis corresponds to $\alpha=\pi / 2$ and $0<\beta<\pi$ a variable angle corresponding to the pitchangle in the Bouligand structure. For this case (Figure 2F), the (110) reflection will consist of two nodes separated by $\pi$ in the vertical axis when the fibre is at rightangles to the beam $(\beta=\pi / 2$; Figure $1 \mathbf{F}$, top), but will be a uniform ring when the fibre is along the beam ( $\beta=0$ or $\pi$; Figure 1F, bottom), with broadened nodes closer 
than $\pi$ for intermediate $\beta$. If the Bouligand layer is not exactly at the $(\alpha=\pi / 2, \beta=$ $\pi / 2$ ) angular position, small deviations will lead to small shifts in the two nodes (upper and lower quadrants) as well as change in the separation between the nodes $^{32,34}$; some simulations of $\mathrm{I}(\chi)$ for different $\alpha, \beta$ are shown in Supplementary Information Figure S6. Simulations of the integrated (110) intensity over a narrow sector $90-95^{\circ}$ versus tilt angle $\beta$ for the idealized state $(\alpha=\pi / 2$ and $0<\beta<\pi)$ show a close positive correlation between intensity and $\beta$. As the Bouligand lamellar structure is characterized by the $0-\pi$ rotation in $\beta$ tilt angle, this correlation justifies the link between (110) intensity and lamellar structure drawn in Figure 2. 


\section{Acknowledgements}

This work was supported by National Key R\&D Program of China (2018YFC0309800), One-hundred Talents Program (Y851552) of Chinese Academy of Science and Strategic Priority Research Program of Chinese Academy of Science (XDB 37000000), and the National Science Foundation for Young Scientists of China (Grant No. 12005253). HSG thanks the BBSRC (BB/R003610/1) and UKRI (MR/R025673/1) for research grant funding. YW thanks the China Scholarship Council for funding. The main synchrotron experiments were carried out at the light source PETRA III (P06 beamline) at DESY, a member of the Helmholtz Association (HGF). We thank Diamond Light Source for the award of beamtime (SM9893-1) for part of the synchrotron experiments.

\section{Contributions}

YZ and HSG conceived and designed the study with input from JG and GF. YZ, YW and HSG performed all experiments. YW provided samples for study. YZ worked with JG and JTR to design and manufacture the compression tester and control software. YZ carried out data reduction with input from JG. YZ, JG, GF, YW, NT, YD and HSG analyzed the data. YZ, YD and HSG wrote the manuscript.

Supporting Information. 2D WAXD patterns, experimental measurements of hydration effects, X-ray transmission, diffraction intensity variations with angular 
rotation, mechanics and ultrastructure from multiple samples, 3D diffraction model formulae and model simulations of X-ray peak positions and intensities. 


\section{References:}

(1) Suksangpanya, N.; Yaraghi, N. A.; Kisailus, D.; Zavattieri, P. Twisting Cracks in Bouligand Structures. J. Mech. Behav. Biomed. Mater. 2017, 76, 38-57.

(2) Yaraghi, N. A.; Guarin-Zapata, N.; Grunenfelder, L. K.; Hintsala, E.; Bhowmick, S.; Hiller, J. M.; Betts, M.; Principe, E. L.; Jung, J. Y.; Sheppard, L.; Wuhrer, R.; McKittrick, J.; Zavattieri, P. D.; Kisailus, D. A Sinusoidally Architected Helicoidal Biocomposite. Adv. Mater. 2016, 28, 6835-6844.

(3) Guarín-Zapata, N.; Gomez, J.; Yaraghi, N.; Kisailus, D.; Zavattieri, P. D. Shear Wave Filtering in Naturally-Occurring Bouligand Structures. Acta Biomater. 2015, 23, 1120.

(4) Tertuliano, O. A.; Greer, J. R. The Nanocomposite Nature of Bone Drives Its Strength and Damage Resistance. Nat. Mater. 2016, 15, 1195-1202.

(5) Raabe, D.; Sachs, C.; Romano, P. The Crustacean Exoskeleton as an Example of a Structurally and Mechanically Graded Biological Nanocomposite Material. Acta Mater. 2005, 53, 4281-4292.

(6) Zimmermann, E. A.; Gludovatz, B.; Schaible, E.; Dave, N. K. N.; Yang, W.; Meyers, M. A.; Ritchie, R. O. Mechanical Adaptability of the Bouligand-Type Structure in Natural Dermal Armour. Nat. Commun. 2013, 4, 2634. https://doi.org/10.1038/ncomms3634 
(7) Yang, W.; Chen, I. H.; Mckittrick, J.; Meyers, M. A. Flexible Dermal Armor in Nature. JOM 2012, 64, 475-485.

(8) Grunenfelder, L. K.; Suksangpanya, N.; Salinas, C.; Milliron, G.; Yaraghi, N.;

Herrera, S.; Evans-Lutterodt, K.; Nutt, S. R.; Zavattieri, P.; Kisailus, D. Bio-Inspired Impact-Resistant Composites. Acta Biomater. 2014, 10, 3997-4008.

(9) Grunenfelder, L. K.; Milliron, G.; Herrera, S.; Gallana, I.; Yaraghi, N.; Hughes, N.; Evans-Lutterodt, K.; Zavattieri, P.; Kisailus, D. Ecologically Driven Ultrastructural and Hydrodynamic Designs in Stomatopod Cuticles. Adv. Mater. 2018, 30, 1705295.

(10) Liu, P.; Duan, H. G.; Lich, L. V; Ye, T.; Bui, T. Q. Buckling of Stomatopod-DactylClub-Inspired Functional Gradient Plates: A Numerical Study. Compos. Struct. 2019, 207, 801-815.

(11) Weaver, J. C.; Milliron, G. W.; Miserez, A.; Evans-Lutterodt, K.; Herrera, S.; Gallana, I.; Mershon, W. J.; Swanson, B.; Zavattieri, P.; DiMasi, E.; Kisailus, D. The Stomatopod Dactyl Club: A Formidable Damage-Tolerant Biological Hammer. Science. 2012, 336, 1275-1280.

(12) Taylor, J. R. A.; Patek, S. N. Ritualized Fighting and Biological Armor: The Impact Mechanics of the Mantis Shrimp's Telson. J. Exp. Biol. 2010, 213, 3496-3504.

(13) Sachs, C.; Fabritius, H.; Raabe, D. Influence of Microstructure on Deformation Anisotropy of Mineralized Cuticle from the Lobster Homarus Americanus. J. Struct. 
Biol. 2008, 161, 120-132.

(14) Sachs, C.; Fabritius, H.; Raabe, D. Hardness and Elastic Properties of Dehydrated Cuticle from the Lobster Homarus Americanus Obtained by Nanoindentation. $J$. Mater. Res. 2006, 21, 1987-1995.

(15) Sachs, C.; Fabritius, H.; Raabe, D. Experimental Investigation of the Elastic-Plastic Deformation of Mineralized Lobster Cuticle by Digital Image Correlation. J. Struct. Biol. 2006, 155, 409-425.

(16) Fabritius, H. O.; Sachs, C.; Triguero, P. R.; Raabe, D. Influence of Structural Principles on the Mechanics of a Biological Fiber-Based Composite Material with Hierarchical Organization: The Exoskeleton of the Lobster Homarus Americanus. Adv. Mater. 2009, 21, 391-400.

(17) Chen, P.-Y.; Lin, A. Y.-M.; McKittrick, J.; Meyers, M. A. Structure and Mechanical Properties of Crab Exoskeletons. Acta Biomater. 2008, 4, 587-596.

(18) Chen, C. J.; Song, J. W.; Zhu, S. Z.; Li, Y. J.; Kuang, Y. D.; Wan, J. Y.; Kirsch, D.; Xu, L. S.; Wang, Y. B.; Gao, T. T.; Wang, Y. L.; Huang, H.; Gan, W. T.; Gong, A.; Li, T.; Xie, J.; Hu, L. B. Scalable and Sustainable Approach toward Highly Compressible, Anisotropic, Lamellar Carbon Sponge. Chem 2018, 4, 544-554.

(19) Schutt, F.; Signetti, S.; Kruger, H.; Roder, S.; Smazna, D.; Kaps, S.; Gorb, S. N.; Mishra, Y. K.; Pugno, N. M.; Adelung, R. Hierarchical Self-Entangled Carbon 
Nanotube Tube Networks. Nat. Commun. 2017, 8, 1215.

https://doi.org/10.1038/s41467-017-01324-7.

(20) Gao, H. L.; Zhu, Y. B.; Mao, L. B.; Wang, F. C.; Luo, X. S.; Liu, Y. Y.; Lu, Y.; Pan, Z.; Ge, J.; Shen, W.; Zheng, Y. R.; Xu, L.; Wang, L. J.; Xu, W. H.; Wu, H. A.; Yu, S. H. Super-Elastic and Fatigue Resistant Carbon Material with Lamellar Multi-Arch Microstructure. Nat. Commun. 2016, 7, 12920. https://doi.org/10.1038/ncomms12920.

(21) Yeni, Y. N.; Schaffler, M. B.; Gibson, G.; Fyhrie, D. P. Prestress Due to Dimensional Changes Caused by Demineralization: A Potential Mechanism for Microcracking in Bone. Ann. Biomed. Eng. 2002, 30, 217-225.

(22) Freeman, J. W.; Silver, F. H. The Effects of Prestrain and Collagen Fibril Alignment on In Vitro Mineralization of Self-Assembled Collagen Fibers. Connect. Tissue Res. $\mathbf{2 0 0 5}, 46,107-115$.

(23) Rausch, M. K.; Kuhl, E. On the Effect of Prestrain and Residual Stress in Thin Biological Membranes. J. Mech. Phys. Solids 2013, 61, 1955-1969.

(24) Nikolov, S.; Fabritius, H.; Petrov, M.; Friák, M.; Lymperakis, L.; Sachs, C.; Raabe, D.; Neugebauer, J. Robustness and Optimal Use of Design Principles of Arthropod Exoskeletons Studied by ab Initio-Based Multiscale Simulations. J. Mech. Behav. Biomed. Mater. 2011, 4, 129-145.

(25) Madi, K.; Staines, K. A.; Bay, B. K.; Javaheri, B.; Geng, H.; Bodey, A. J.; Cartmell, 
S.; Pitsillides, A. A.; Lee, P. D. In Situ Characterization of Nanoscale Strains in Loaded Whole Joints via Synchrotron X-Ray Tomography. Nat. Biomed. Eng. 2020, $4,343-354$.

(26) Stock, S. R.; De Carlo, F.; Almer, J. D. High Energy X-Ray Scattering Tomography Applied to Bone. J. Struct. Biol. 2008, 161, 144-150.

(27) Ludwig, W.; Reischig, P.; King, A.; Herbig, M.; Lauridsen, E. M.; Johnson, G.; Marrow, T. J.; Buffiere, J. Y. Three-Dimensional Grain Mapping by X-Ray Diffraction Contrast Tomography and the Use of Friedel Pairs in Diffraction Data Analysis. Rev. Sci. Instrum. 2009, 80, 033905.

(28) Larson, B. C.; Yang, W.; Ice, G. E.; Budai, J. D.; Tischler, J. Z. Three-Dimensional XRay Structural Microscopy with Submicrometre Resolution. Nature 2002, 415, 887890.

(29) Simons, H.; King, A.; Ludwig, W.; Detlefs, C.; Pantleon, W.; Schmidt, S.; Stohr, F.; Snigireva, I.; Snigirev, A.; Poulsen, H. F. Dark-Field X-Ray Microscopy for Multiscale Structural Characterization. Nat. Commun. 2015, 6, 6098. https://doi.org/10.1038/ncomms7098.

(30) Pfeiffer, F. X-Ray Ptychography. Nat. Photonics 2018, 12, 9-17.

(31) Zhang, Y.; De Falco, P.; Wang, Y.; Barbieri, E.; Paris, O.; Terrill, N. . J.; Falkenberg, G.; Pugno, N. M.; Gupta, H. S. Towards In Situ Determination of 3D Strain and 
Reorientation in the Interpenetrating Nanofibre Networks of Cuticle. Nanoscale 2017, 9, 11249-11260.

(32) Zhang, Y.; Paris, O.; Terrill, N. J.; Gupta, H. S. Uncovering Three-Dimensional Gradients in Fibrillar Orientation in an Impact-Resistant Biological Armour. Sci. Rep. 2016, 6, 26249. https://doi.org/10.1038/srep26249.

(33) Liebi, M.; Georgiadis, M.; Kohlbrecher, J.; Holler, M.; Raabe, J.; Usov, I.; Menzel, A.; Schneider, P.; Bunk, O.; Guizar-Sicairos, M. Small-Angle X-Ray Scattering Tensor Tomography: Model of the Three-Dimensional Reciprocal-Space Map, Reconstruction Algorithm and Angular Sampling Requirements. Acta Crystallogr. Sect. A Found. Adv. 2018, A74, 12-24.

(34) Stribeck, N. On the Determination of Fiber Tilt Angles in Fiber Diffraction. Acta Crystallogr. Sect. A Found. Crystallogr. 2009, A65, 46-47.

(35) Grünewald, T. A.; Liebi, M.; Wittig, N. K.; Johannes, A.; Sikjaer, T.; Rejnmark, L.; Gao, Z.; Rosenthal, M.; Guizar-Sicairos, M.; Birkedal, H. Mapping the 3D Orientation of Nanocrystals and Nanostructures in Human Bone: Indications of Novel Structural Features. Sci. Adv. 2020, 6, eaba4171.

(36) Ogawa, Y.; Hori, R.; Kim, U. J.; Wada, M. Elastic Modulus in the Crystalline Region and the Thermal Expansion Coefficients of Alpha-Chitin Determined Using Synchrotron Radiated X-Ray Diffraction. Carbohyd. Polym. 2011, 83, 1213-1217. 
(37) Rojob, H.; El-Hacha, R. Self-Prestressing Using Iron-Based Shape Memory Alloy for Flexural Strengthening of Reinforced Concrete Beams. Aci Struct. J. 2017, 114, 523532.

(38) Compton, B. G.; Lewis, J. A. 3D-Printing of Lightweight Cellular Composites. Adv. Mater. 2014, 26, 5930-5935.

(39) Huang, W. J.; Dai, K.; Zhai, Y.; Liu, H.; Zhan, P. F.; Gao, J. C.; Zheng, G. Q.; Liu, C. T.; Shen, C. Y. Flexible and Lightweight Pressure Sensor Based on Carbon Nanotube/Thermoplastic Polyurethane-Aligned Conductive Foam with Superior Compressibility and Stability. ACS Appl. Mater. Interfaces 2017, 9, 42266-42277.

(40) Tadayon, M.; Amini, S.; Masic, A.; Miserez, A. The Mantis Shrimp Saddle: A Biological Spring Combining Stiffness and Flexibility. Adv. Funct. Mater. 2015, 25, $6437-6447$.

(41) Romano, P.; Fabritius, H.; Raabe, D. The Exoskeleton of the Lobster Homarus Americanus as an Example of a Smart Anisotropic Biological Material. Acta Biomater. 2007, 3, 301-309.

(42) Wittig, N. K.; Palle, J.; Østergaard, M.; Frølich, S.; Birkbak, M. E.; Spiers, K. M.; Garrevoet, J.; Birkedal, H. Bone Biomineral Properties Vary across Human Osteonal Bone. ACS Nano 2019, 13, 12949-12956.

(43) Liebi, M.; Georgiadis, M.; Menzel, A.; Schneider, P.; Kohlbrecher, J.; Bunk, O.; 
Guizar-Sicairos, M. Nanostructure Surveys of Macroscopic Specimens by SmallAngle Scattering Tensor Tomography. Nature 2015, 527, 349-352.

(44) Almer, J. D.; Stock, S. R. Internal Strains and Stresses Measured in Cortical Bone via High-Energy X-Ray Diffraction. J. Struct. Biol. 2005, 152, 14-27.

(45) Masic, A.; Bertinetti, L.; Schuetz, R.; Chang, S.-W.; Metzger, T. H.; Buehler, M. J.;

Fratzl, P. Osmotic Pressure Induced Tensile Forces in Tendon Collagen. Nat.

Commun. 2015, 6, 5942. https://doi.org/10.1038/ncomms6942.

(46) Bertinetti, L.; Masic, A.; Schuetz, R.; Barbetta, A.; Seidt, B.; Wagermaier, W.; Fratzl,

P. Osmotically Driven Tensile Stress in Collagen-Based Mineralized Tissues. J. Mech.

Behav. Biomed. Mater. 2015, 52, 14-21.

(47) Inamdar, S. R.; Knight, D. P.; Terrill, N. J.; Karunaratne, A.; Cacho-Nerin, F.; Knight, M. M.; Gupta, H. S. The Secret Life of Collagen: Temporal Changes in Nanoscale Fibrillar Pre-Strain and Molecular Organization during Physiological Loading of Cartilage. ACS Nano 2017, 11, 9728-9737.

(48) Inamdar, S. R.; Barbieri, E.; Terrill, N. J.; Knight, M. M.; Gupta, H. S. Proteoglycan Degradation Mimics Static Compression by Altering the Natural Gradients in Fibrillar Organisation in Cartilage. Acta Biomater. 2019, 97, 437-450.

(49) Wang, Y.; Zhang, Y.; Terrill, N. J.; Barbieri, E.; Pugno, N. M.; Gupta, H. S. MatrixInduced Pre-Strain and Mineralization-Dependent Interfibrillar Shear Transfer Enable 
3D Fibrillar Deformation in a Biogenic Armour. Acta Biomater. 2019, 100, 18-28.

(50) Al-Sawalmih, A.; Li, C. H.; Siegel, S.; Fratzl, P.; Paris, O. On the Stability of Amorphous Minerals in Lobster Cuticle. Adv. Mater. 2009, 21, 4011-4015.

(51) Gupta, H. S.; Seto, J.; Wagermaier, W.; Zaslansky, P.; Boesecke, P.; Fratzl, P. Cooperative Deformation of Mineral and Collagen in Bone at the Nanoscale. Proc. Natl. Acad. Sci. U. S. A. 2006, 103, 17741-17746.

(52) Zimmermann, E. A.; Schaible, E.; Bale, H.; Barth, H. D.; Tang, S. Y.; Reichert, P.; Busse, B.; Alliston, T.; Ager, J. W.; Ritchie, R. O.; Ager 3rd, J. W.; Ritchie, R. O. Age-Related Changes in the Plasticity and Toughness of Human Cortical Bone at Multiple Length Scales. Proc. Natl. Acad. Sci. U. S. A. 2011, 108, 14416-14421.

(53) Yao, H.; Gao, H. Multi-Scale Cohesive Laws in Hierarchical Materials. Int. J. Solids Struct. 2007, 44, 8177-8193.

(54) Li, M. Y.; Carter, R.; Douglas, A.; Oakes, L.; Pint, C. L. Sulfur Vapor-Infiltrated 3D Carbon Nanotube Foam for Binder-Free High Areal Capacity Lithium-Sulfur Battery Composite Cathodes. ACS Nano 2017, 11, 4877-4884.

(55) Gui, X. C.; Wei, J. Q.; Wang, K. L.; Cao, A. Y.; Zhu, H. W.; Jia, Y.; Shu, Q. K.; Wu, D. H. Carbon Nanotube Sponges. Adv. Mater. 2010, 22, 617-621.

(56) Saba, N.; Jawaid, M.; Alothman, O. Y.; Paridah, M. T. A Review on Dynamic Mechanical Properties of Natural Fibre Reinforced Polymer Composites. Constr. 
Build. Mater. 2016, 106, 149-159.

(57) Ashiotis, G.; Deschildre, A.; Nawaz, Z.; Wright, J. P.; Karkoulis, D.; Picca, F. E.;

Kieffer, J. The Fast Azimuthal Integration Python Library: PyFAI. J. Appl.

Crystallogr. 2015, 48, 510-519.

(58) Grunewald, T. A.; Rennhofer, H.; Tack, P.; Garrevoet, J.; Wermeille, D.; Thompson,

P.; Bras, W.; Vincze, L.; Lichtenegger, H. C.; Grünewald, T. A.; Rennhofer, H.; Tack,

P.; Garrevoet, J.; Wermeille, D.; Thompson, P.; Bras, W.; Vincze, L.; Lichtenegger,

H. C. Photon Energy Becomes the Third Dimension in Crystallographic Texture

Analysis. Angew. Chem. Int. Ed. 2016, 55, 12190-12194. 